\title{
What makes a difference: A method to determine whether a change in an image affects the perceived gist
}

\author{
XANDRA VAN MONTFORT \\ Technische Universiteit Eindhoven, The Netherlands
}

\begin{abstract}
Although many changes in images and real-world situations can go undetected, researchers have suggested that changes affecting the gist of an image are likely to be noticed. This article proposes a method to determine whether a change in an image affects its gist. The method was designed to determine a systematical difference between sets of descriptions of subjectively interpretable images. First, for each image, a set of descriptions is generated. Then, a rater judges good fit or poor fit for the descriptions for each image, providing the base for categorization. Finally, a Fisher exact probability test is used to determine a difference between sets of descriptions. The method is applied to determine a gist change for 18 test sets. The proposed method makes it possible to compare subjective interpretations of images, while leaving room for differences in interpretation of an image by different people.
\end{abstract}

Would you notice the difference between an image of a happy couple and an image of a vampire attempting to bite a young woman (Figure 1)? Research on change detection has revealed that a lot of changes can go unnoticed (see, e.g., Simons \& Rensink, 2005, for an overview). Researchers have suggested for many years that changes not affecting the gist of an image are unlikely to be detected (Friedman, 1979; Simons \& Levin, 1997). Wolfe (1998) rephrased this suggestion by asking whether changes affecting the gist are sufficient for efficient change detection. The problem that arises in answering this question is how to determine whether a change to an image affects its gist.

The gist of a scene is usually referred to as a short description capturing the essence or identity of the scene (see, e.g., Hollingworth \& Henderson, 2002; Rensink, 2000 ) or the central theme (Potter, 1975). When gist is mentioned in relation to what is remembered of a scene, it is defined as the activated schema or frame, including unexpected aspects (Biederman, 1981; Friedman, 1979). Rensink, O'Regan, and Clark (1997) suggested that a description of the most interesting aspects of an image is included in the gist. All aspects mentioned above are likely to be part of the gist. There is, however, another important aspect: Gist will depend on the observer. People interpret a scene on the basis of what they see (image properties) and what they know (knowledge, experience, interests, expectations). The identification of the essence of a scene, the activated schema or frame, the interesting aspects of a scene - and, therefore, the gist — can differ for different people.

Knowing the exact interpretation of a particular image by different people is not relevant for research on the ef- fects of gist on change detection. For instance, Figure 1B can be interpreted by different people as either a vampire, Dracula, or a man dressed up for Halloween. Although different people may have different interpretations of Figures $1 \mathrm{~A}$ and $1 \mathrm{~B}$, most people will agree that Figures $1 \mathrm{~A}$ and 1B have a different gist. When investigating the effects of gist on change detection, the question that needs to be answered is not "How should we define gist," but instead "How can we determine a change in gist?"

The gist is affected by a change when people systematically interpret the original image differently than the changed version of the image. The images in Figure 1, for instance, are likely to be interpreted differently as a result of the added fangs in the second image. It is, however, not always this clear whether two images are interpreted differently. Showing someone the two images simultaneously and asking whether they interpret them differently is not sufficient. When a person sees both images side by side, the difference can become salient; that is, an area not receiving attention when an image is presented alone might receive attention when two images are presented together. For instance, people might only notice the fangs in Figure 1B because they are searching for a difference between the two images. If the fangs do not attract attention when Figure 1B is presented alone, the image would not be interpreted differently from Figure 1A. Simply asking people whether a change affects the interpretation may therefore lead to overestimating the relevance of the change for the interpretation of the image.

Asking a person to interpret both images and then comparing the two interpretations is also not sufficient to determine a difference in interpretation between two

X.van Montfort, xandra_van_montfort@hotmail.com 


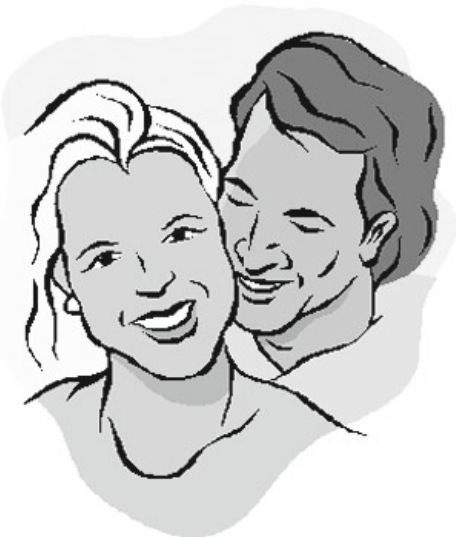

A

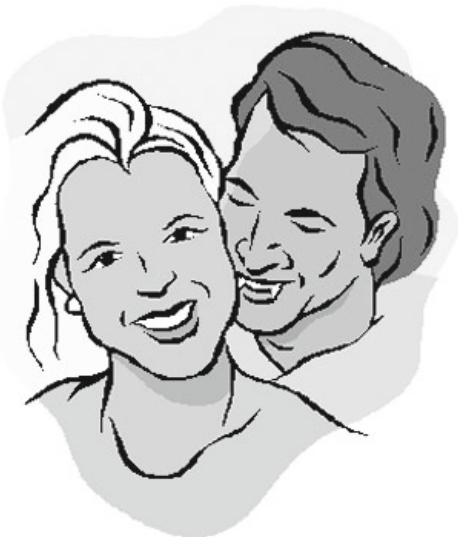

B

Figure 1. Example of a change affecting the gist of the image. The added fangs result in different interpretations of Images $1 \mathrm{~A}$ and $1 \mathrm{~B}$.

images. When a person is asked to interpret two images, the interpretation of the second image is influenced by the first image (Garner, 1974). This again may lead to overestimating the importance of the difference.

It is better to ask a person to interpret only one image. Asking one person to interpret one image and another person to interpret the other image complicates the comparison of interpretations. Since two people might differ in their interpretation of one image, a different interpretation of the two images might be a result of individual interpretation differences or of the differences between the two images. Con- sider the descriptions in Table 1 - which were generated by people who were asked to provide their interpretation of either Figure 2A or 2B - to get an idea of the challenge that a comparison of subjective interpretations poses.

To determine whether Figures $2 \mathrm{~A}$ and $2 \mathrm{~B}$ are systematically interpreted differently, the descriptions of Figure 2A have to be compared with those of Figure 2B. One possible method for comparing the descriptions in Table 1 would be to categorize them according to their semantic content. Generally, a categorization made on the basis of the content of the descriptions would require an extensive coding

Table 1

Descriptions Generated by Participants Who

Were Asked for Their Interpretation of Either Figure 2A or Figure 2B

Descriptions for Figure 2A

A woman with an injury on her left leg is assisted at the counter of a shop, while a man is opening the door for her

In a hospital the receptionist gives a letter to a patient

A woman with crutches gets an envelope and has to leave the room

Waiting room of the general practitioner

Check-up at the doctor's

Colleagues are happy that colleague is back at work, only she is not happy, probably because of her injury

Hospital

The desk at a family doctor's practice

A woman gets a letter at the reception of an office

Joann with her broken leg gets redundancy pay

A patient gets a letter in the hospital

A woman is being assisted

A woman with a broken leg receives an envelope from a woman behind the counter (maybe in the hospital)

People at the office kindly converse with the disabled woman

Descriptions for Figure 2B

Someone receives a letter and then has to leave

A desk clerk who hands over an envelope to a woman

Activity at the reception

A woman gets a letter from a woman behind a desk and there are two men, one of whom opens the door for her

At the office a man opens the door for a woman

It is busy at the office

A woman gets an invitation

Getting fired

An office where something is handed over to a woman in front of a counter

People who are working at an office

Two men see a beautiful woman and look at her

Woman delivering an envelope to another woman 


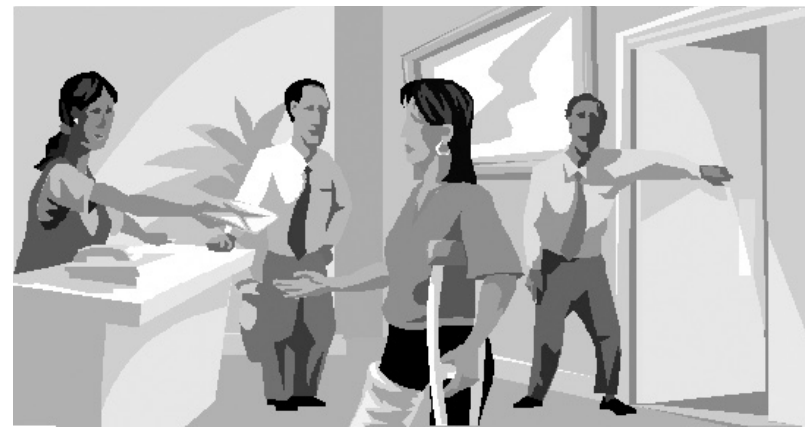

A

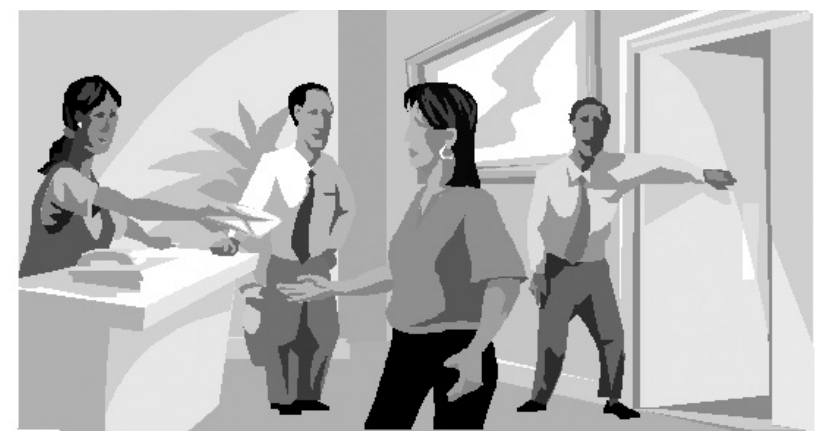

B

Figure 2. Example of a change possibly affecting the gist of the image. The deleted cast and crutch could result in different interpretations of Images $2 \mathrm{~A}$ and $2 \mathrm{~B}$.

scheme with one category for every possible interpretation of the image and (potentially complex) rules for mapping descriptions onto categories. More importantly, the coding scheme is prone to researcher bias. The categories that the researcher decides to include in the coding scheme could bias the interpretation of the descriptions by the person coding them in the direction of the researcher's hypotheses. For instance, the choice of included categories dictates whether descriptions mentioning "a patient" should be categorized differently than those mentioning "a woman." Most likely, the coding scheme will be developed - or at least adjusted - after the collection of descriptions, since it is very difficult to anticipate every possible interpretation. Another disadvantage of a categorization made on the basis of the semantic content of the descriptions is that each set of images requires a new coding scheme.

In this article, an alternative method for categorizing and comparing descriptions is proposed. In the proposed method, only one criterion is used as the base for the categorization: An interpretation either fits or does not fit an image. The method makes it easier to determine whether two images are systematically interpreted differently. This makes it possible to apply the method for selecting images for studies on the effect of gist changes.

\section{Proposed Method}

A systematic difference between interpretations of images can be determined in three steps. In the first step for each image, a set of descriptions is generated. In the second step, a rater judges a "good fit" or a "poor fit" for the descriptions of the images, providing the base for categorization. In the third step, the categorized descriptions are analyzed statistically. The method is illustrated by applying it to the three circles of different sizes shown in Figure 3.

Step 1: Collecting descriptions, the generator task. The first step in determining if images are interpreted differently is to have human participants generate descriptions reflecting their interpretations. Because seeing more than one of the images could influence the way an image is described (Garner, 1974), for each image, a separate group of people (the generators) is asked to provide the descriptions. For the circle example, 10 hypo- thetical descriptions of each of the circles from Figure 3 are presented in Table 2. Since Circles A and B are both described as being "large" and "small" about half of the time, it seems fair to say that the sets of descriptions of Circles A and B are similar. Circle C, on the other hand, is only described as being "small." The descriptions of Circle $\mathrm{C}$ can be said to be different from the descriptions of Circles A and B. A difference or similarity in sets of descriptions is not always as obvious, which should be clear from the descriptions presented in Table 1 for the images from Figure 2. Like the descriptions in Table 1, the descriptions in Table 2 represent subjective interpretations of the images. Therefore, there is no qualitative difference between the descriptions; that is, there are no right or wrong descriptions.

Step 2: Categorizing descriptions, the rater task. In the second step, participants (the raters) who are not involved in generating the descriptions, and are naive to the nature of the task, judge the descriptions. Each description is judged on the basis of whether the description could be an appropriate interpretation ("good fit," 1) or a less appropriate interpretation ("poor fit," 0 ) for each of the im-

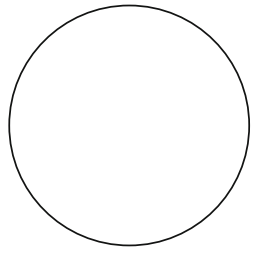

Circle A
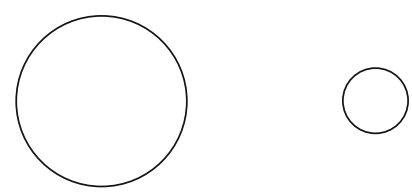

Circle B

\section{Circle C}

Figure 3. Three different-sized circles for illustration of the method to determine a systematic difference between sets of descriptions.

Table 2

Frequency of Hypothetical Descriptions "Small" and "Large" for the Three Different-Sized Circles in Figure 3

\begin{tabular}{cccc}
\hline Description & Circle A & Circle B & Circle C \\
\hline Small & 4 & 5 & 10 \\
Large & 6 & 5 & - \\
\hline
\end{tabular}


ages. The rater reads all descriptions and views all images. The rater has no knowledge about which description was generated for which image. The rater's task results in a fit answer for each description for each image.

Considering the circles in Figure 3 and their descriptions, there are four logical ${ }^{1}$ views a rater could have concerning the appropriateness of the descriptions. A rater could view all three circles as being large (hypothetical Rater 1), or all three circles as being small (hypothetical Rater 2). Alternatively, a rater could consider Circles A and $\mathrm{B}$ both to be large and Circle $\mathrm{C}$ to be small (hypothetical Rater 3), or Circle A to be large and both Circles B and C as being small (hypothetical Rater 4). The interpretation of the rater is reflected in the assigned fit answers for each description for each image. For instance, hypothetical Rater 1 will judge all "small" descriptions to be a poor fit (0) for all three circles, and all "large" descriptions to be a good fit (1) for each circle. In Table 3, the fit answers for the descriptions "small" and "large" matching the interpretations of the four hypothetical raters are displayed.

In the same way that one could conclude that there is no qualitative difference between the descriptions, one could also conclude that there is no qualitative difference in interpretations by the rater; that is, there is no right or wrong interpretation of the descriptions by the rater. All four interpretations - as displayed in Table 3 - are equally valid, and they should lead to the same conclusions about a systematic difference between sets of descriptions. Therefore, a conclusion on a systematic difference between sets of descriptions cannot be made on the basis of the fit answers alone. For example, if a conclusion would be made on the basis of whether a description would fit equally to Circle A and B, the interpretation of hypothetical Rater 4 would lead to a different conclusion than the interpretations of hypothetical Raters 1,2, and 3. The fit answers contain information about how a rater interprets the images, whereas the objective of the method was to compare sets of descriptions.

Step 3: Comparing sets of descriptions, the researcher's task. In order to determine whether the sets of descriptions of images are systematically different, the fit answers are combined to represent a fit pattern. When comparing the descriptions of Circle A and B, each description can be denoted as a fit pattern, combining the fit answers for Circles A and B. There are four possible fit patterns for each description.

(00) The description is not appropriate for either Circle A or Circle B.

(10) The description is appropriate for Circle A, but is not appropriate for Circle B.

(01) The description is not appropriate for Circle A, but appropriate for Circle B.

(11) The description is appropriate for both Circle A and Circle B.

A decision on a difference between sets of descriptions is not made on the basis of the observed fit patterns. A frequent occurrence of the fit patterns " 10 " and " 01 " is not necessarily proof of a difference between sets of descriptions of the images. The fit patterns " 10 " and " 01 " indicate that the rater interprets the two images differently.
Table 3

Fit Answers for Descriptions "Small" and "Large" for the Three Circles in Figure 3, Matching the Interpretations of Four Hypothetical Raters

\begin{tabular}{|c|c|c|c|c|c|c|c|c|c|c|c|c|}
\hline \multirow[b]{2}{*}{ Description } & \multicolumn{3}{|c|}{$\begin{array}{c}\text { Rater } 1 \\
(\mathrm{~A}+\mathrm{B}+ \\
\mathrm{C} \text { Large }) \\
\end{array}$} & \multicolumn{3}{|c|}{$\begin{array}{c}\text { Rater } 2 \\
(\mathrm{~A}+\mathrm{B}+ \\
\mathrm{C} \text { Small }) \\
\end{array}$} & \multicolumn{3}{|c|}{$\begin{array}{c}\text { Rater } 3 \\
\text { (A + B Large, } \\
\text { C Small) } \\
\end{array}$} & \multicolumn{3}{|c|}{$\begin{array}{c}\text { Rater } 4 \\
\text { (A Large, } \\
\text { B + C Small }\end{array}$} \\
\hline & $\overline{\mathrm{A}}$ & B & $\bar{C}$ & $\overline{\mathrm{A}}$ & B & $\mathrm{C}$ & $\mathrm{A}$ & $\mathrm{B}$ & $\mathrm{C}$ & $\mathrm{A}$ & $\mathrm{B}$ & $\mathrm{C}$ \\
\hline Small & 0 & 0 & 0 & 1 & 1 & 1 & 0 & 0 & 1 & 0 & 1 & 1 \\
\hline Large & 1 & 1 & 1 & 0 & 0 & 0 & 1 & 1 & 0 & 1 & 0 & 0 \\
\hline
\end{tabular}

Hypothetical Rater 4 in Table 3 illustrates that the occurrence of fit patterns " 10 " and " 01 " does not necessarily mean a difference between sets of descriptions. Although hypothetical Rater 4 discriminates between Circles A and $\mathrm{B}$, this does not affect the similarity between the sets of descriptions of Circles A and B.

The source of a description (Circles A, B, or C) should be considered to find a systematic difference in occurring fit patterns between the sets of descriptions. Table 4 contains the hypothetically occurring fit patterns for the descriptions of Circles A, B, and C. The columns of this table represent the source of the descriptions (Circle A and $\mathrm{B}$ in Table 4A and Circles A and C in Table 4B). The rows represent the fit patterns $(00,10,01$, and 11$)$. In the cells, the number of descriptions that were appointed to the fit pattern is displayed.

If there is a systematic difference in the way images are described, then there should be a difference between the fit patterns for the associated sets of descriptions. When the fit patterns assigned to the descriptions of one image differ from those assigned to the descriptions of another image, it indicates a difference between the sets of descriptions. A difference does not depend on which fit patterns occur. In the circle example, the different interpretations of the hypothetical raters resulted in different fit patterns. Note that this should not affect the conclusion, since all raters have seen the same descriptions and all rater interpretations are equally valid. Thus, it is not the fit patterns, but the distribution over the fit patterns that determines whether there is a systematic difference between sets of descriptions. Statistical analysis will reveal whether two fit-pattern distributions are significantly different.

\section{Statistical Analysis}

As was already suggested by the hypothetical data in Table 4, in reality, several fit patterns occur rarely, or not at all. Conventional tests for contingency tables (such as a chi-square test) will be unreliable or produce no results at all, with sparsely filled tables. For this case, a FisherFreeman-Halton exact probability test for contingency tables can be used (Freeman \& Halton, 1951). The exact test calculates the sum of probabilities of the specific table and all patterns that are "more extreme." If the sum of these probabilities is less than .05 , then we conclude that there is a significant difference between the fit-pattern distributions for descriptions of two images. We may then conclude that this result indicates a systematic difference between sets of descriptions. An analysis of fit-pattern distributions for the circle example in Table 4A reveals no difference 
Table 4A

Frequency of Hypothetically Occurring Fit Patterns for Descriptions of Circles A and B for Four Hypothetical Raters

\begin{tabular}{|c|c|c|c|c|c|c|c|c|}
\hline \multirow[b]{3}{*}{ Fit Pattern } & \multicolumn{8}{|c|}{ Source of Descriptions (Circle A and Circle B) } \\
\hline & \multicolumn{2}{|c|}{$\begin{array}{c}\text { Rater } 1 \\
(\mathrm{~A}+\mathrm{B}+ \\
\text { C Large })\end{array}$} & \multicolumn{2}{|c|}{$\begin{array}{c}\text { Rater } 2 \\
(\mathrm{~A}+\mathrm{B}+ \\
\mathrm{C} \text { Small })\end{array}$} & \multicolumn{2}{|c|}{$\begin{array}{c}\text { Rater } 3 \\
(\mathrm{~A}+\mathrm{B} \text { Large, } \\
\text { C Small })\end{array}$} & \multicolumn{2}{|c|}{$\begin{array}{c}\text { Rater } 4 \\
\text { (A Large, } \\
\text { B + C Small) }\end{array}$} \\
\hline & $\mathrm{A}$ & B & $\mathrm{A}$ & $\mathrm{B}$ & A & $\mathrm{B}$ & A & B \\
\hline 00 & 4 & 5 & 6 & 5 & 4 & 5 & - & - \\
\hline 10 & - & - & - & - & - & - & 6 & 5 \\
\hline 01 & - & - & - & - & - & - & 4 & 5 \\
\hline 11 & 6 & 5 & 4 & 5 & 6 & 5 & - & - \\
\hline
\end{tabular}

Table 4B

Frequency of Hypothetically Occurring Fit Patterns for Descriptions of Circles $A$ and $C$ for Four Hypothetical Raters Source of Descriptions (Circle A and Circle C) \begin{tabular}{llll}
\hline Rater 1 & Rater 2 & Rater 3 &
\end{tabular} $(\mathrm{A}+\mathrm{B}+(\mathrm{A}+\mathrm{B}+\quad(\mathrm{A}+\mathrm{B}$ Large, $\quad$ (A Large, C Large) C Small) $\quad$ C Small) $\quad$ B + C Small)

\begin{tabular}{|c|c|c|c|c|c|c|c|c|}
\hline \multirow[b]{2}{*}{ Fit Pattern } & & & & \\
\hline & $\mathrm{A}$ & $\mathrm{C}$ & A & $\mathrm{C}$ & A & $\mathrm{C}$ & A & $\mathrm{C}$ \\
\hline 00 & 4 & 10 & 6 & - & - & - & - & - \\
\hline 10 & - & - & - & - & 6 & - & 6 & - \\
\hline 01 & - & - & - & - & 4 & 10 & 4 & 10 \\
\hline 11 & 6 & - & 4 & 10 & - & - & - & - \\
\hline
\end{tabular}

between the fit-pattern distributions for the descriptions of Circles A and B ( $p=1.0$ for all four hypothetical raters). Analysis of the data in Table $4 \mathrm{~B}$ reveals a difference between the fit-pattern distributions in the descriptions of Circles A and C ( $p=.011$ for all four hypothetical raters). On the basis of these results, we conclude that Circles A and $\mathrm{B}$ are described in a similar way and that Circle $\mathrm{C}$ is described in a different way than Circle A.

\section{Validity}

Next, the validity of the proposed method and some of its benefits and potential drawbacks are considered. Finding a significant difference between fit-pattern distributions when sets of descriptions of two images are similar is unlikely. As demonstrated in the circle example, even if a rater interprets Circles A and B as being different (hypothetical Rater 4), this does not lead to a different conclusion for a difference between the sets of descriptions. The only way a rater can produce fit patterns indicating a difference in descriptions - if there is in fact no difference - is when a rater is extremely lucky in guessing the source of a description and judges accordingly. In the circles example, this would mean that a rater would discriminate between descriptions denoted as "large" generated for Circle A, and descriptions denoted as "large" generated for Circle B. Not only is it unlikely that a rater would be able to make this distinction, it also means that the rater would be inconsistent, since he or she needs to assign different fit answers for identical descriptions. The chance of finding a significant difference between two fitpattern distributions when there is, in fact, no difference in descriptions (Type I error) is small.

The chance of finding no difference between fit-pattern distributions when sets of descriptions of two images are different is relatively large (Type II error). The method is conservative. Raters have to judge whether a description would be appropriate for an image. A rater with a lot of imagination might feel that all descriptions are appropriate for each image (e.g., descriptions "large" and "small" are equally appropriate for all three circles). In this case, such a liberal interpretation would result in all " 11 " fit patterns, resulting in no difference between fit-pattern distributions. Similarly, a rater lacking imagination might conclude that none of the descriptions is appropriate, resulting in all " 00 " fit patterns. In such extreme cases, even when one circle is consistently described as "small" and the other described as "large," no difference between fitpattern distributions will be found. Consequently, when the fit-pattern distributions are not significantly different, this does not imply that the descriptions are the same. The method will detect a gist change if it is clear to both generators and raters that there is a difference.

The method becomes more reliable with more descriptions for each image. With more descriptions per image, the interpretation of a larger group of people is represented, making it more likely that the set of descriptions reflects the general interpretation of an image for a particular target group. Also, with more descriptions, a less common interpretation of an image will have correspondingly less weight in the final analysis. The minimum amount of descriptions generated for each image to assess a difference in interpretation between two images may vary with the complexity of the images. Complex or ambiguous images may require more descriptions than images displaying a univocal scene. Although the necessary amount of descriptions was not assessed directly, the recommended amount ranges between 10 and 20 descriptions for each image.

Asking several raters to judge the descriptions can increase confidence for the researcher. When using more than one rater, the data from each rater is analyzed separately. As demonstrated in the circle example, the fit patterns provided by the raters do not need to be the same to reach the same conclusion. Differences can occur when raters have varying interpretations. When different raters reach different conclusions, this can be the result of three reasons. First, a rater might have been extremely lucky in guessing and has produced fit patterns indicating a difference even though the sets of descriptions do not differ (Type I error). Second, a rater might not share the interpretations of the generators (Type II error). Third, the sets of descriptions may not be sufficiently different. When the difference between sets of descriptions is small, different fit patterns for only a few descriptions can result in different conclusions for different raters. Because the chance that a rater produces data indicating a difference in descriptions when there is in fact no difference in descriptions is small, the chance that two independent raters produce such data for the same sets of descriptions is negligible. Therefore, when two raters produce significant differences, there is evidence that sets of descriptions are systematically different. Since the chance of finding no difference between fit-pattern distributions when, in fact, the sets of descriptions are different is relatively large, using three raters is recommended. When the data from 
two out of three raters produces a significant outcome, it indicates a gist change. For an even more conservative approach, agreement between all three raters can be applied as criterion.

\section{METHOD}

The method was applied to determine whether a change intended by the researcher to affect the gist of an image was perceived as such by the target group. Images used to establish this included 18 sets of colored Microsoft clipart images. Each set consisted of the original image and two altered versions. One alteration was intended to change the gist (similar to Figures $1 \mathrm{~B}$ and $2 \mathrm{~B}$ ); the other alteration was intended not to change the gist (such as a swap of hair color that could be realized in the first image of Figure 1).

Step 1: Collecting descriptions, the generator task. The generators were 213 students from the University of Utrecht and the Radboud University Nijmegen (73 male, 140 female, mean age of 20.6 years). The generator task was part of a set of unrelated tasks performed by each participant. Because of time limitations, each participant was asked to describe only five images instead of one image from each of the 18 sets of images. ${ }^{2}$ Each generator saw one of the three versions of an image. The image was shown on a computer screen for $5 \mathrm{sec}$. The generators were then asked to write down their interpretation of what the image was about. After completion, the next image was shown for $5 \mathrm{sec}$, until each generator had described five images. For each image, between 10 and 16 descriptions were generated. ${ }^{3}$

Step 2: Categorizing descriptions, the rater task. The descriptions were rated by three staff members (one female, mean age of 31.3 years) who were naive to the nature of the task. Each rater received a booklet containing 18 rating tasks (one task per set of images). The rating task consisted of all three images side by side, followed by all descriptions of these images in random order. The raters were told that the people who provided the descriptions had been asked to describe their interpretation of the image. The raters were instructed to look at the images, notice the difference, and determine for each description whether it was a good fit or a poor fit for each of the images. We stressed that the rater should consider whether someone could possibly have provided the description and not whether the description exactly reflected the rater's personal interpretation. ${ }^{4}$

Step 3: Comparing sets of descriptions, the researcher's task. The fit answers for the three sets of descriptions were collected for each of the 18 sets of images. The original image was labeled Image A; the image that was altered with the intention of changing the gist was labeled Image $B$, and the image that was altered with the intention of not changing the gist was labeled Image $C$. The fit patterns were analyzed for each rater separately. This tactic resulted in six contingency tables for each set of images (Image B was not compared with Image C). Each contingency table was analyzed using the FisherFreeman-Halton exact probability test.
As an example, the contingency tables for the descriptions from Table 1 for the images in Figure 2 are shown in Table 5. Notice that Rater 3 clearly demonstrated a different interpretation than the other two raters, which we can conclude from the frequencies in the " 11 " and " 01 " cells for the descriptions of Image 2B. For all three raters, there is a difference in fit-pattern distributions $(p<.001)$, indicating a systematic difference in the descriptions of Images $A$ and $B$ in Figure 2. On the basis of these results, we conclude that the deletion of the cast and crutch does affect the way that the images are described, and therefore the gist (of the image).

For the 18 sets of images, a comparison between Image $\mathrm{A}$ and Image $\mathrm{B}$ (intended gist change) shows that 11 sets yield a significant difference $(p<.05)$ for all three raters. One set of images showed a significant difference for two raters, but no significant difference for the third. Two sets of images showed a significant difference for only one rater. Four sets of images showed no significant difference for any rater. Comparing Image $\mathrm{A}$ and Image $\mathrm{C}$ (no gist change intended) did not show any significant difference for any of the raters for all 18 sets of images. On the basis of the results, we may conclude that the 12 sets of images - with a significant outcome for the intended gist change image for at least two out of three raters and no significant outcome for any of the raters for the image in which no gist change was intended-would be suitable for use in further experiments.

\section{DISCUSSION}

The intended gist change did not always result in significant differences in fit-pattern distributions for all three raters. Two examples of sets of images in which the intended gist change was not found are discussed below.

Figure 4 displays an example of images in which the intended gist change did not result in fit-pattern distributions that were different for any of the raters. The descriptions of Images 4A and 4B were not systematically different. Examination of the descriptions revealed that a large majority of generators did not mention the wheelchair, suggesting that it was not relevant for the interpretation of Image 4B. Since the generators did not mention the wheelchair, the raters were not able to distinguish between the descriptions provided for Images $4 \mathrm{~A}$ and $4 \mathrm{~B}$. It is possible that for generators from a different target group-for instance, elderly or disabled people - the wheelchair in Figure 4B would be relevant for the interpretation of the image and would subsequently be mentioned more often

Table 5

Frequency of Occurring Fit Patterns for Descriptions of Images 2A and 2B for Three Raters

\begin{tabular}{|c|c|c|c|c|c|c|}
\hline \multirow[b]{3}{*}{ Fit Pattern } & \multicolumn{6}{|c|}{ Source of Descriptions } \\
\hline & \multicolumn{2}{|c|}{ Rater 1} & \multicolumn{2}{|c|}{ Rater 2} & \multicolumn{2}{|c|}{ Rater 3} \\
\hline & $2 \mathrm{~A}$ & $2 \mathrm{~B}$ & $2 \mathrm{~A}$ & $2 \mathrm{~B}$ & $2 \mathrm{~A}$ & $2 B$ \\
\hline 00 & 2 & 3 & 2 & - & 2 & - \\
\hline 10 & 11 & - & 10 & - & 10 & - \\
\hline 01 & 1 & 9 & 2 & 11 & - & 3 \\
\hline 11 & - & - & - & 1 & 2 & 9 \\
\hline
\end{tabular}




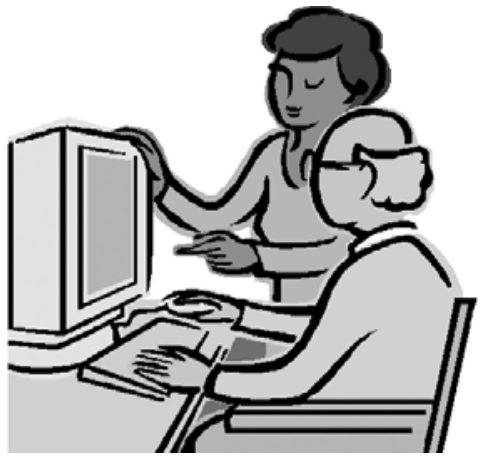

A

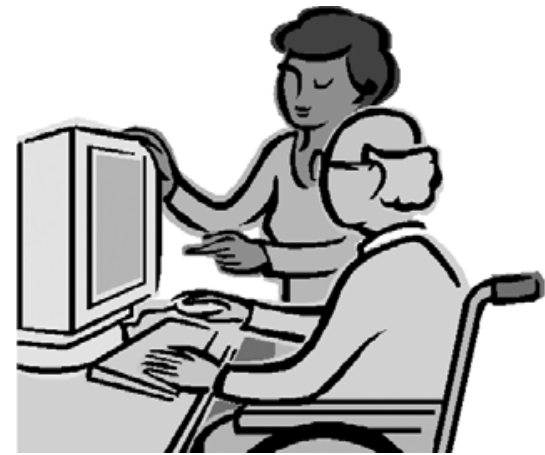

B

Figure 4. Example of a change that did not affect the gist. The addition of the wheelchair features did not result in systematical differences between descriptions of Images $4 A$ and $4 B$.

in the descriptions, resulting in systematical differences between descriptions of Images 4A and 4B.

Figure 5 displays an example of images in which the intended gist change resulted in fit-pattern distributions that were different for only one rater. In this case, the descriptions provided by the generators were not as unequivocal as those of Figure 4. For both images in Figure 5, descriptions were generated that did mention Paris or France and descriptions were generated that did not mention location. This result indicates that not all generators found the Eiffel Tower important for the interpretation of the image. The variance in the importance of the Eiffel Tower seen with the generators can also be seen in the judgments of the raters. One rater judged all descriptions to fit both images, regardless of whether the description mentioned Paris or France. Presumably, this rater was familiar with
Paris and recognized the scene in Figure 5B as a typical Parisian scene, even without the Eiffel Tower in the back. This interpretation did not result in a significant difference in fit-pattern distributions. The other two raters did differentiate between descriptions that mentioned location and those that did not. If the generators would have systematically described Figure 5A as a Parisian scene and Figure $5 \mathrm{~B}$ as not, such a strategy would have resulted in a difference in fit-pattern distributions. Because of the limited difference between descriptions of the two images (i.e., the sets of descriptions were not sufficiently different), a small difference in interpretation between the two raters resulted in a significant difference between distributions for one rater, but not for the other. Again, it is possible that other generators - for instance, Americans who are not as familiar with Paris-would have inter-

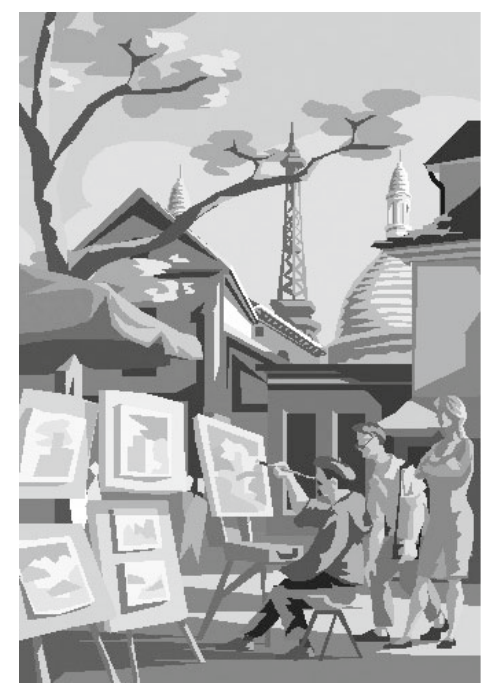

A

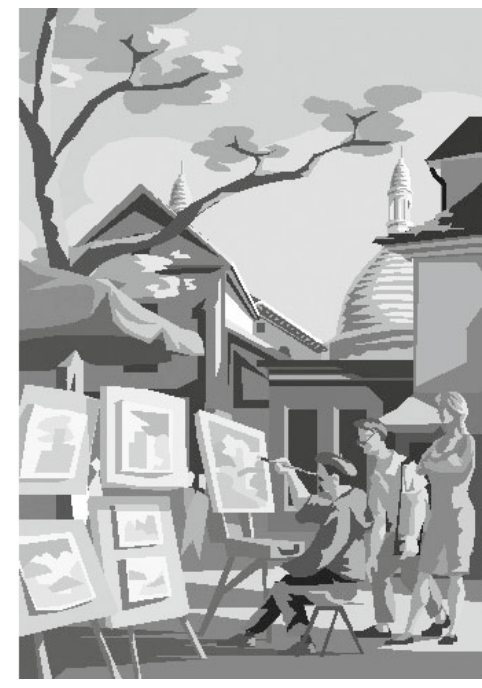

B

Figure 5. Example of a change that did not affect the gist. The deletion of the Eiffel Tower did not result in sufficiently different descriptions for Images 5A and 5B. Two out of three raters produced fit-pattern distributions that did not differ significantly. 
preted and described the images differently, resulting in different conclusions.

\section{Conclusion and Implications}

In this article, a method is proposed to determine whether a change in an image affects the gist of that image. The gist of an image is reflected in a verbal description of a subjective interpretation on what the image is about. To determine a gist change, subjective interpretations of the original image need to be compared with interpretations of the altered version of the image. The method overcomes some of the problems that are associated with categorizing and comparing sets of subjective interpretations. Only one criterion is used as the base for the categorization: An interpretation either fits or does not fit the image. We conclude that sets of descriptions are systematically different if there is a difference between fit-pattern distributions for two sets of descriptions.

The proposed method makes it possible to compare subjective interpretations of images, while leaving room for differences in the interpretation of an image by different people. In comparison with categorizing descriptions on the basis of their semantic content, the proposed method of categorizing on the basis of fit patterns has two advantages. First, the categorization is not prone to researcher bias, since the researcher does not need to define categories. Second, the fixed categories make the method applicable to all kinds of images without the difficulty of coming up with extensive coding schemes.

A legitimate concern is that a gist change as determined with the proposed method need not necessarily reflect a gist change for everyone. Since knowledge, experience, interests, and expectations influence the interpretation of images, descriptions will reflect the subjective interpretations for a particular group of generators. Strictly speaking, the proposed method makes it possible to determine whether a particular target group - in this case, Dutch university students-would perceive a change as affecting the gist of an image. However, this does not greatly limit the applicability of the method. If this method is used to select images for further research, such as research on the effect of gist change on image recognition, the target group in the ensuing studies should be identical to that used for the image selection.

The method described is not limited to the detection of gist changes in images. By comparing the results from the method for two different target groups-for instance, experts and novices - a possible difference in image interpretation can be found. At a more general level, the method might be applicable to determine any significant difference in subjectively interpretable stimuli (taste, smell, or even associations with brand names). The (only) two conditions that need to be fulfilled are that a description of the interpretation of the stimuli can be generated, and that these descriptions can be judged by one or more raters as "likely" or "not likely" descriptions of that stimulus.

\section{AUTHOR NOTE}

The research was supported by the Netherlands Organization for Scientific Research. The author thanks Paul de Greef for fruitful discussions and Jan van Bolhuis for help with the statistical analysis. Address correspondence to X. van Montfort, University of Glasgow, Department of Psychology, 58 Hillhead Street, Glasgow G12 8QB, Scotland (e-mail: xandra_van_montfort@hotmail.com).

\section{REFERENCES}

Biederman, I. (1981). On the semantics of a glance at a scene. In M. Kubovy \& J. R. Pomerantz (Eds.), Perceptual organization (pp. 213-253). Hillsdale, NJ: Erlbaum.

Freeman, G. H., \& Halton, J. H. (1951). Note on an exact treatment of contingency goodness-of-fit and other problems of significance. Biometrika, 38, 141-149.

Friedman, A. (1979). Framing pictures: The role of knowledge in automatized encoding and memory for gist. Journal of Experimental Psychology: General, 108, 316-355.

GARNER, W. R. (1974). The processing of information and structure. Potomac, MD: Erlbaum.

Hollingworth, A., \& Henderson, J. M. (2002). Accurate visual memory for previously attended objects in natural scenes. Journal of Experimental Psychology: Human Perception \& Performance, 28, 113-136.

Potter, M. C. (1975). Meaning in visual search. Science, 187, 965-966.

Rensink, R. A. (2000). The dynamic representation of scenes. Visual Cognition, 7, 17-42.

Rensink, R. A., O'Regan, J. K., \& Clark, J. J. (1997). To see or not to see: The need for attention to perceive changes in scenes. Psychological Science, 8, 368-373.

Simons, D. J., \& Levin, D. T. (1997). Change blindness. Trends in Cognitive Sciences, 1, 261-267.

Simons, D. J., \& Rensink, R. A. (2005). Change blindness: Past, present, and future. Trends in Cognitive Sciences, 9, 16-20.

Wolfe, J. M. (1998). Visual memory: What do you know about what you saw? Current Biology, 8, 303-304.

\section{NOTES}

1. Other views are possible, such as perceiving Circle A and B "small" and Circle C "large." These other options and their corresponding fit answers will lead to the same conclusions as the discussed options.

2. The minimum required amount of participants depends on the preferred amount of descriptions for each image and the number of versions of each image. Without the time limitation, the current study could have been conducted with as little as 30 participants (three groups of 10 participants each describing one of the three versions of each of the 18 images).

3. Some of the images described by the generators are not part of the research reported here.

4. Although the method was designed to handle different interpretations by different people, it is possible that a rater's personal interpretation is not reflected in the generated descriptions. If a rater would judge the descriptions only on the basis of his or her own personal interpretation, then the chance of finding no difference between sets of descriptions increases. If, for instance, a rater would interpret the images in Figure 2 as "a man being courteous," instructing a rater to judge the appropriateness of the descriptions only on the basis of his or her own interpretation would result in "poor fit" judgments for all descriptions that do not mention the polite man. On the basis of the results, one would then conclude that there is no difference between descriptions.

(Manuscript received October 18, 2005; revision accepted for publication April 1, 2006.) 